\title{
On the projectivity of the moduli spaces of curves
}

\author{
M. D. T. Cornalba ${ }^{1}$ \\ Dipartimento di Matematica \\ Università di Pavia
}

1. There are basically three algebraic proofs of the projectivity of the moduli spaces of stable curves: the original one by Knudsen [8][9], the proof by Mumford [12] and Gieseker [5], which makes heavy use of the machinery of geometric invariant theory, and the more recent one by Viehweg [15] and Kollàr [10], which relies instead on the semipositivity of the direct images of powers of the relative dualizing sheaf and, at least in Kollàr's version, does not use geometric invariant theory at all. In this note, combining ideas from the above papers and from [4], we shall outline a proof of projectivity which, we believe, is simpler and more direct than any of the existing ones. However, while the proofs by Mumford, Gieseker, Viehweg, and Kollàr are applicable, at least in principle, to a wide variety of moduli problems, ours uses in an essential way the peculiarities of the problem at hand, and it is hard to see how it could be extended to other situations.

Mumford's idea is to use the machinery of geometric invariant theory to directly construct the moduli space of stable curves as a projective quotient of the Chow or Hilbert scheme of pluricanonically embedded stable curves by the action of the projective linear group. To be able to do so, one needs to show that the $k$-canonical images of stable curves are stable in the invariant-theoretic sense for high $k$. This is quite well understood, although not really easy, for smooth curves; one notices that $k$-canonically embedded stable curves are linearly stable, and then proves that, for smooth curves, linear stability implies invariant-theoretic stability. In the singular case, by contrast, both Mumford and Gieseker have to rely on indirect arguments which are quite long and involved. Our main point is that one can avoid proving the invariant-theoretic stability of singular stable curves provided one is willing to grant that moduli space exists as a complete algebraic space; that this is the case, incidentally, is relatively easy to prove. As in [10], we prove projectivity by applying to a suitable invertible sheaf one of the standard numerical ampleness criteria (Seshadri's criterion in our case). The necessary numerical estimates are obtained using the techniques of [4]. More precisely, the invariant-theoretic stability of linearly stable smooth curves is used to prove an inequality for families of stable curves which, suitably interpreted, says that the hypothesis of Seshadri's criterion is satisfied for curves in moduli which are not contained in the boundary. The case of curves lying in the boundary is then reduced to the previous one by standard elementary techniques; in order to properly carry out this procedure, which can be viewed as a sort of induction on the genus, it is convenient to deal simultaneously with the moduli spaces of stable $n$-pointed curves as well. In a sense, then, our approach is to use invariant-theoretic stability as a means of obtaining numerical inequalities, rather than as a step in the construction of quotients by group actions.

\footnotetext{
${ }^{1}$ Research supported in part by the M.U.R.S.T. national project "Geometria Algebrica".
} 
2. Let $\bar{M}_{g, n}$ be the moduli space of stable $n$-pointed curves of genus $g$. It is not too hard to prove that it is a reduced algebraic space (see [3] or [13], for instance) and then the stable reduction theorem shows it is complete and separated. We shall produce an ample line bundle on $\bar{M}_{g, n}$; in order to describe it we need to briefly recall a few facts about the Picard groups of moduli spaces, referring to [12], [6], and [2] for more details. The rational Picard group $\operatorname{Pic}\left(\bar{M}_{g, n}\right) \otimes \mathbb{Q}$ contains three distinguished classes $\lambda, \delta$, and $\psi$; this last class is zero when $n=0$. These classes do not correspond to ordinary line bundles, but only to fractional ones. If we are given a family $F$ of stable $n$-pointed curves of genus $g$, consisting of a family $f: X \rightarrow S$ of noded curves together with sections $\sigma_{1}, \ldots, \sigma_{n}$, and $\gamma: S \rightarrow \bar{M}_{g, n}$ is the corresponding map to moduli, the classes $\lambda, \delta$, and $\psi$ pull back via $\gamma$ to classes $\lambda_{F}$, $\delta_{F}$, and $\psi_{F}$ on $S$, which are easily described in geometric terms. The class $\lambda_{F}$ is the class of the line bundle $\operatorname{det}\left(f_{*} \omega_{f}\right)$, where $\omega_{f}$ is the relative dualizing sheaf $\omega_{X / S}$, while, at least when the general fiber of $f$ is smooth, $\delta_{F}$ is the class of $\mathcal{O}_{S}(D)$, where $D$ is the divisor in $S$ parametrizing singular fibers. As for $\psi_{F}$, it is defined as $\sum \psi_{i, F}$, where $\psi_{i, F}$ is the class of $\sigma_{i}^{*}\left(\omega_{f}\right)$. In particular, we see that $\lambda_{F}, \delta_{F}$, and $\psi_{F}$ correspond to ordinary, and not fractional, line bundles. When no confusion is likely, we shall often write $\lambda_{f}, \delta_{f}$, and $\psi_{f}$ instead of $\lambda_{F}, \delta_{F}$, and $\psi_{F}$. It will be convenient to give slightly different descriptions of $\lambda_{F}$ and $\psi_{F}$. Set $C_{i}=\sigma_{i}(S)$ : we claim that $\lambda_{F}$ is the class of $\operatorname{det}\left(f_{*}\left(\omega_{f}\left(\sum C_{i}\right)\right)\right)$ and that $\psi_{i, F}$ is the class of $\sigma_{i}^{*}\left(\mathcal{O}_{X}\left(-C_{i}\right)\right)$. If $n=0$, there is nothing to prove. If $n>0$, the residue homomorphism $\omega_{f}\left(C_{i}\right) \rightarrow \mathcal{O}_{C_{i}}$ is onto and has $\omega_{f}$ as kernel. This means that $\sigma_{i}^{*}\left(\omega_{f}\left(C_{i}\right)\right)$ is trivial, that is, that $\sigma_{i}^{*}\left(\omega_{f}\right)$ and $\sigma_{i}^{*}\left(\mathcal{O}_{X}\left(-C_{i}\right)\right)$ are isomorphic. It also implies that there is an exact sequence

$$
0 \rightarrow \omega_{f} \rightarrow \omega_{f}\left(\sum C_{i}\right) \stackrel{R e s}{\longrightarrow} \sum \mathcal{O}_{C_{i}} \rightarrow 0
$$

where Res is the residue homomorphism. Taking direct images and noticing that

$$
R^{1} f_{*}\left(\omega_{f}\right)=\mathcal{O}_{S}, \quad R^{1} f_{*}\left(\omega_{f}\left(\sum C_{i}\right)\right)=0
$$

one gets another exact sequence

$$
0 \rightarrow f_{*} \omega_{f} \rightarrow f_{*}\left(\omega_{f}\left(\sum C_{i}\right)\right) \rightarrow \mathcal{O}_{S}^{n} \rightarrow \mathcal{O}_{S} \rightarrow 0
$$

Thus

$$
\operatorname{det}\left(f_{*}\left(\omega_{f}\left(\sum C_{i}\right)\right)\right)=\operatorname{det}\left(f_{*} \omega_{f}\right) \operatorname{det}\left(\mathcal{O}_{S}^{n}\right) \operatorname{det}\left(\mathcal{O}_{S}\right)^{-1}=\operatorname{det}\left(f_{*} \omega_{f}\right)
$$

as desired.

It is not hard to show that there is a finite morphism

$$
\zeta: Z \rightarrow \bar{M}_{g, n}
$$

where $Z$ is a scheme, with the additional property that there exists on $Z$ a family of stable $n$-pointed curves

$$
\xi: \mathcal{X} \rightarrow Z, \quad \tau_{1}, \ldots, \tau_{n}: Z \rightarrow \mathcal{X}
$$

such that, for any $z \in Z$, the moduli point of $\left(\xi^{-1}(z), \tau_{1}(z), \ldots, \tau_{n}(z)\right)$ is $\zeta(z)$; for a proof, we refer to [10]. To show that a line bundle $\mathcal{L}$ on $\bar{M}_{g, n}$ is ample, it will then suffice to show 
that $\zeta^{*}(\mathcal{L})$ is ample on $Z$, and to do this it will be enough to check that the hypotheses of Seshadri's criterion of ampleness (cf. [7]) are satisfied. In other words, what we'll have to check is that there is a positive constant $\alpha$ such that, for any reduced and irreducible complete curve $S \subset Z$,

$$
\operatorname{deg} \zeta^{*}(\mathcal{L})_{\mid S} \geq \alpha m(S)
$$

where $m(S)$ stands for the maximum of the multiplicities of points of $S$.

We shall prove ampleness for the line bundle on $\bar{M}_{g, n}$ corresponding to the class $12 \lambda-\delta+\psi$. As any subscheme $S$ of $Z$ yields, by pullback, a family of stable curves over $S$, Seshadri's criterion says that it suffices to prove the following result.

THEOREM (2.2). Let $g$ and $n$ be non-negative integers; assume moreover that $g>1$ if $n=0$ and that $g>0$ if $n=1$ or $n=2$. Then there is a positive constant $\alpha$ such that, for any non-isotrivial family $F$ of stable $n$-pointed curves of genus $g$ over a reduced and irreducible complete curve $S$,

$$
12 \operatorname{deg}\left(\lambda_{F}\right)-\operatorname{deg}\left(\delta_{F}\right)+\operatorname{deg}\left(\psi_{F}\right) \geq \alpha m(S)
$$

where $m(S)$ is the maximum multiplicity of points of $S$.

In this section we shall show that it suffices to prove Theorem (2.2) for families whose general fiber is smooth. Let $F$ be as in (2.2); say it consists of a family $f: X \rightarrow S$ of noded curves of genus $g$, together with sections $\sigma_{1}, \ldots, \sigma_{n}$. Suppose the general fiber of $f$ is singular. Denote by $N(f)$ the union of the one-dimensional components of the locus of the nodes of the fibers of $f$. After a finite base change $N(f)$ becomes a union of sections. Since the number of singular points of a stable $n$-pointed curve of genus $g$ is bounded, the degree of the base change in question can also be bounded; as the effect of a base change of degree $d$ on the invariants $\operatorname{deg} \lambda_{F}, \operatorname{deg} \delta_{F}$, and $\operatorname{deg} \psi_{F}$ is to multiply them by $d$, we are then reduced to proving (2.2) under the hypothesis that $N(f)$ is a union of sections. This means that $f: X \rightarrow S$ can be obtained from a family $f^{\prime}: X^{\prime} \rightarrow S$ of noded curves whose general fiber is smooth, but not necessarily connected, with disjoint sections $\sigma_{1}, \ldots, \sigma_{n}, \tau_{1}, \ldots, \tau_{2 l}$, by identifiying $\tau_{2 j-1}(S)$ to $\tau_{2 j}(S)$, for $j=1, \ldots, l$. Let $\pi: X^{\prime} \rightarrow X$ be the identification map, $X_{1}, \ldots, X_{m}$ the connected components of $X^{\prime}$, and, for each $i$, let $f_{i}: X_{i} \rightarrow S$ be the restriction of $f^{\prime}$ to $X_{i}$. Since $f: X \rightarrow S$ is a family of stable $n$-pointed curves, each $f_{i}: X_{i} \rightarrow S$, together with the sections $\sigma_{v}$ and $\tau_{j}$ that lie on $X_{i}$, whose number we denote by $n_{i}$, is a family of stable $n_{i}$-pointed curves. Set $C_{v}=\sigma_{v}(S), D_{j}=\tau_{j}(S)$. Consider the map $R$ from $\pi_{*}\left(\omega_{f^{\prime}}\left(\sum C_{v}+\sum D_{j}\right)\right)$ to $\mathcal{O}_{N(f)}$ which, along each section $\pi\left(D_{2 j}\right)$, associates to a meromorphic relative differential along $f^{\prime}$ the sum of its residues along $D_{2 j-1}$ and along $D_{2 j}$. This map fits into an exact sequence

$$
0 \rightarrow \omega_{f}\left(\sum C_{v}\right) \rightarrow \pi_{*}\left(\omega_{f^{\prime}}\left(\sum C_{v}+\sum D_{j}\right)\right) \stackrel{R}{\longrightarrow} \mathcal{O}_{N(f)} \rightarrow 0
$$

Since in our situation

$$
R^{1} f_{*}\left(\pi_{*}\left(\omega_{f^{\prime}}\left(\sum C_{v}+\sum D_{j}\right)\right)\right)=R^{1} f_{*}^{\prime}\left(\omega_{f^{\prime}}\left(\sum C_{v}+\sum D_{j}\right)\right)=0
$$


this in turn yields an exact sequence

$$
0 \rightarrow f_{*}\left(\omega_{f}\left(\sum C_{v}\right)\right) \rightarrow f_{*}^{\prime}\left(\omega_{f^{\prime}}\left(\sum C_{v}+\sum D_{j}\right)\right) \rightarrow f_{*}\left(\mathcal{O}_{N(f)}\right) \rightarrow R^{1} f_{*}\left(\omega_{f}\left(\sum C_{v}\right)\right) \rightarrow 0
$$

Since $f_{*}\left(\mathcal{O}_{N(f)}\right)=\mathcal{O}_{S}^{l}$, while $R^{1} f_{*}\left(\omega_{f}\left(\sum C_{v}\right)\right)$ is zero for $n>0$ and equals $\mathcal{O}_{S}$ when $n=0$, we conclude that

$$
\operatorname{deg}\left(\lambda_{f}\right)=\sum_{i=1}^{m} \operatorname{deg}\left(\lambda_{f_{i}}\right)
$$

On the other hand, one has

$$
\operatorname{deg}\left(\delta_{f}\right)=\sum_{i=1}^{m} \operatorname{deg}\left(\delta_{f_{i}}\right)+\sum_{j=1}^{2 l}\left(D_{j} \cdot D_{j}\right)
$$

(cf. [6]), while

$$
\sum_{i=1}^{m} \operatorname{deg}\left(\psi_{f_{i}}\right)=-\sum_{v=1}^{n}\left(C_{v} \cdot C_{v}\right)-\sum_{j=1}^{2 l}\left(D_{j} \cdot D_{j}\right)=\operatorname{deg}\left(\psi_{f}\right)-\sum_{j=1}^{2 l}\left(D_{j} \cdot D_{j}\right) .
$$

This implies that

$$
\operatorname{deg}\left(\psi_{f}\right)-\operatorname{deg}\left(\delta_{f}\right)=\sum_{i=1}^{m} \operatorname{deg}\left(\psi_{f_{i}}\right)-\sum_{i=1}^{m} \operatorname{deg}\left(\delta_{f_{i}}\right)
$$

and hence, taking into account (2.3), that

$$
12 \operatorname{deg}\left(\lambda_{f}\right)-\operatorname{deg}\left(\delta_{f}\right)+\operatorname{deg}\left(\psi_{f}\right)=\sum_{i=1}^{m}\left(12 \operatorname{deg}\left(\lambda_{f_{i}}\right)-\operatorname{deg}\left(\delta_{f_{i}}\right)+\operatorname{deg}\left(\psi_{f_{i}}\right)\right) .
$$

Thus it suffices to prove Theorem (2.2) when the general fiber of $f$ is smooth.

3. In this section we shall prove (2.2) for families whose general fiber is smooth, under the additional hypothesis that $n=0$. As we have announced, we shall use the techniques of [4]. The results of that paper deal with families of curves and line bundles, or, more precisely, with families $f: X \rightarrow S$ of noded curves plus a line bundle $L$ on $X$; at times we shall refer to such a setup, perhaps somewhat improperly, as a family of polarized noded curves. We shall say that a family of polarized noded curves is generically trivial if a general point $s \in S$ has a neighbourhood $U$ such that $f^{-1}(U) \rightarrow U$ is isomorphic to a product family $X_{0} \times U \rightarrow U$ and, moreover, the restriction of $L$ to $f^{-1}(U)$ comes, by pullback, from a line bundle on $X_{0}$.

Consider a family $f: X \rightarrow S$ of connected noded curves of genus $g$ over a reduced and irreducible complete curve $S$, and let $L$ be a line bundle on $X$ of relative degree $d$; suppose 
the general fiber of $f$ is smooth and that $r=\operatorname{dim} H^{0}\left(f^{-1}(s), L_{\mid f^{-1}(s)}\right)$ is independent of $s \in S$. The main result of [4] says that

$$
r(L \cdot L) \geq 2 d \operatorname{deg} f_{*}(L),
$$

provided that

a) $L$ is relatively ample;

b) for general $s \in S$ the restriction $L_{\mid f^{-1}(s)}$ is base-point-free, very ample, and embeds $f^{-1}(s)$ as a (Hilbert) semi-stable subscheme of projective space.

Let's review the proof. For large $h$ the map

$$
\varphi: \operatorname{Sym}^{h}\left(f_{*} L\right) \rightarrow f_{*}\left(L^{h}\right)
$$

is onto. Let $N$ be the rank of $f_{*}\left(L^{h}\right)$, and consider the map

$$
\wedge^{N} \varphi: \wedge^{N} \operatorname{Sym}^{h}\left(f_{*} L\right) \rightarrow \operatorname{det}\left(f_{*}\left(L^{h}\right)\right)
$$

The proof is based on the following remark. If $s$ is a point of $S$, choosing bases for $H^{0}\left(f^{-1}(s), L_{\mid f^{-1}(s)}\right)$ and for $\operatorname{det} H^{0}\left(f^{-1}(s), L_{\mid f^{-1}(s)}^{h}\right)$ identifies

$$
\left(\wedge^{N} \varphi\right)_{s}: \wedge^{N} \operatorname{Sym}^{h} H^{0}\left(f^{-1}(s), L_{\mid f^{-1}(s)}\right) \rightarrow \operatorname{det} H^{0}\left(f^{-1}(s), L_{\mid f^{-1}(s)}^{h}\right)
$$

with a point $v \in V$, where $V$ is the $N$-th exterior power of the $h$-th symmetric power of the standard representation of $S L(r)$. To say that $f^{-1}(s)$, embedded by the linear system $\left|L_{\mid f^{-1}(s)}\right|$, is Hilbert semi-stable means that, for large $h, v$ is semi-stable under the action of $S L(r)$. Given an $S L(r)$-invariant polynomial $P \in \operatorname{Sym}^{l} V^{*}$, we may evaluate $P$ at $v$; whether $P$ vanishes at $v$ or not clearly does not depend on the choice of bases, so that we can speak of $P$ vanishing, or not vanishing, at $\left(\wedge^{N} \varphi\right)_{s}$. Now let $s$ be a general point of $S$. If $h$ is sufficiently large, by semi-stability there is an $S L(r)$-invariant homogeneous polynomial $P$ that does not vanish at $\left(\wedge^{N} \varphi\right)_{s}$. Choosing local trivializations for $f_{*} L$ and $\operatorname{det}\left(f_{*}\left(L^{h}\right)\right)$, we get a local regular function $\eta$ on $S$ by evaluating $P$ on $\wedge^{N} \varphi$. Since $P$ is $S L(r)$-invariant, changing trivialization of $f_{*} L$ by a matrix $A$ changes $\eta$ by a factor $(\operatorname{det} A)^{-l N h / r}$, where $l$ is the degree of $P$. Thus if, as we may, we assume $l$ to be of the form $r M$, where $M$ is an integer, this produces a non-zero section of

$$
\operatorname{det}\left(f_{*}\left(L^{h}\right)\right)^{r M} \otimes \operatorname{det}\left(f_{*} L\right)^{-h N M},
$$

so that this line bundle has non-negative degree. One then concludes by evaluating the degree by means of the Grothendieck Riemann-Roch theorem and letting $h$ go to infinity.

We can improve slightly on the conclusion if we modify the hypotheses by requiring that the image of $f^{-1}(s)$ in projective space be stable, and not only semi-stable, that the family we are dealing with be generically non-trivial, and by replacing condition a) above with the requirement that $R^{1} f_{*}\left(L^{h}\right)=0$ for large $h$. Let $s^{\prime}$ be any point of $S$; if $s$ is a general point of $S$ and (3.1) is onto at $s^{\prime}$, then $\left(\wedge^{N} \varphi\right) s^{\prime}$ does not belong to the $G L(r)$ orbit of $\left(\wedge^{N} \varphi\right)_{s}$. Therefore we can choose $P$ so that it vanishes at $\left(\wedge^{N} \varphi\right)_{s^{\prime}}$, and hence we 
get a non-zero section of $\operatorname{det}\left(f_{*}\left(L^{h}\right)\right)^{r M} \otimes \operatorname{det}\left(f_{*} L\right)^{-h N M}$ that vanishes at $s^{\prime}$; this same conclusion is trivially true if (3.1) is not onto at $s^{\prime}$. If $s^{\prime}$ is a point of maximum multiplicity on $S$ this shows that

$$
\operatorname{deg}\left(\operatorname{det}\left(f_{*}\left(L^{h}\right)\right)^{r M} \otimes \operatorname{det}\left(f_{*} L\right)^{-h N M}\right) \geq m(S) .
$$

Notice that, by the compactness of the Hilbert scheme parametrizing subschemes of $\mathbb{P}^{r-1}$ with Hilbert polynomial $p(t)=d t+1-g$, the integers $h$ and $M$ can be chosen independently of $f: X \rightarrow S$ and $L$. On the other hand, the Riemann-Roch theorem yields

$$
\begin{gathered}
N=h d+1-g, \\
\operatorname{deg}\left(f_{*}\left(L^{h}\right)\right)=\frac{h^{2}}{2}(L \cdot L)-\frac{h}{2}\left(L \cdot \omega_{f}\right)+\operatorname{deg} \lambda_{f},
\end{gathered}
$$

so that we get

Lemma (3.2). Let $g \geq 2, d$, and $r$ be positive integers. Then there is a positive integer $h_{0}$ such that for any integer $h \geq h_{0}$ there is a positive integer $M$ with the following property. Consider any generically non-trivial family of polarized noded connected genus $g$ curves $(f: X \rightarrow S, L)$ such that the relative degree of $L$ is $d$. Assume moreover that:

i) $S$ is a reduced and irreducible complete curve and the general fiber of $f$ is smooth,

ii) $R^{1} f_{*}\left(L^{h}\right)=0$ for large $h$ and $\operatorname{dim} H^{0}\left(f^{-1}(s), L_{\mid f^{-1}(s)}\right)=r$ for any $s \in S$,

iii) for general $s \in S$ the restriction $L_{\mid f^{-1}(s)}$ is base-point-free, very ample, and embeds $f^{-1}(s)$ as a (Hilbert) stable subscheme of $\mathbb{P}^{r-1}$.

Then

$$
\begin{aligned}
{\left[\frac{r}{2}(L \cdot L)-d \operatorname{deg}\left(f_{*} L\right)\right] h^{2} } & -\frac{r}{2}\left(L \cdot \omega_{f}\right) h \\
& +r \operatorname{deg}\left(\lambda_{f}\right)+(g-1) \operatorname{deg}\left(f_{*} L\right) \geq \frac{1}{M} m(S) .
\end{aligned}
$$

In practical applications, to check that iii) holds we shall rely on the fact that a smooth curve of genus $g$ embedded in projective space by a complete linear system of degree greater than $2 g$ is linearly stable by Clifford's theorem, and hence, as is proved for instance in [5], stable.

Let's apply Lemma (3.2) to $L=\omega_{f}^{k}$, where $f: X \rightarrow S$ is as in the statement of Theorem (2.2), $n=0$, the general fiber of $f$ is smooth, and $k>2$. Conditions i), ii), and iii) are satisfied, and we have

$$
d=2 k(g-1), \quad r=(2 k-1)(g-1) .
$$

On the other hand, it is proved in [12] that

$$
\begin{gathered}
\left(\omega_{f} \cdot \omega_{f}\right)=12 \operatorname{deg} \lambda_{f}-\operatorname{deg} \delta_{f} \\
\operatorname{deg}\left(f_{*}\left(\omega_{f}^{k}\right)\right)=\operatorname{deg} \lambda_{f}+\frac{k^{2}-k}{2}\left(12 \operatorname{deg} \lambda_{f}-\operatorname{deg} \delta_{f}\right) .
\end{gathered}
$$


Thus expanding the left-hand side of (3.3) yields, for fixed $k$, a polynomial in $h$ whose coefficients are rational linear combinations of $\operatorname{deg} \lambda_{f}$ and $\operatorname{deg} \delta_{f}$ and whose leading term is

$$
h^{2} \frac{k^{2}(g-1)}{2}\left[12 \operatorname{deg} \lambda_{f}-\operatorname{deg} \delta_{f}-\frac{4}{k} \operatorname{deg} \lambda_{f}\right] .
$$

Hence, for large enough $h$, we get an inequality of the form

$$
\beta \operatorname{deg} \lambda_{f}-\operatorname{deg} \delta_{f} \geq \alpha m(S)
$$

where $0<\beta<12$ and $\alpha$ is a positive constant. Since in our situation $\operatorname{deg} \delta_{f}$ is non-negative, this implies that

$$
12 \operatorname{deg} \lambda_{f}-\operatorname{deg} \delta_{f} \geq \alpha m(S),
$$

as desired. Thus (2.2) is proved for families whose general fiber is smooth when $n=0$.

4. In this section we shall complete the proof of (2.2). We begin with a lemma. Consider a family $f: X \rightarrow S$ of noded curves over a reduced and irreducible complete curve $S$. Suppose the general fiber of $f$ is smooth, and that $f$ has a section $C$ which does not pass through any of the singular points of the fibers of $f$. We shall say that an irreducible component of a fiber is exceptional if it is smooth and rational and meets the rest of the fiber only in one point. Let $E_{1}, \ldots, E_{h}$ be exceptional components that intersect $C$, and let $p_{1}, \ldots, p_{h}$ be the points where they meet the rest of their respective fibers. Near $p_{i}$, $X$ is of the form $x y=\eta_{i}$, where $\eta_{i}$ is a function on $S$. The functions $\eta_{i}$ define a Cartier divisor on $S$, whose degree we denote by $\mu$. Let $f^{\prime}: X^{\prime} \rightarrow S$ be the family of noded curves obtained from $f: X \rightarrow S$ by contracting $E_{1}, \ldots, E_{h}$, and let $C^{\prime}$ be the image of $C$ in $X^{\prime}$ : it is a section of $f^{\prime}$ which does not pass through any of the singular points of the fibers of $f^{\prime}$.

Lemma $(4.1) \cdot\left(C^{\prime} \cdot C^{\prime}\right)=(C \cdot C)+\mu$.

The two sides of the equality do not change if we replace $f: X \rightarrow S$ with $X \times_{S} T \rightarrow T$ and $C$ with $C \times{ }_{S} T$, where $T$ is the normalization of $S$. In the proof, then, we may as well assume that $S$ is smooth. By induction, it is also enough to do the case $h=1$; we shall write $E$ for $E_{1}$ and $p$ for $p_{1}$. Near $p, X$ is of the form $x y=t^{k}$, where $t$ is a local parameter on $T$, so that $\mu=k$. If $k>1, X$ is singular at $p$. Desingularizing it has the effect of replacing $p$ with a chain of $k-1$ smooth rational curves $F_{1}, \ldots, F_{k-1}$ of selfintersection -2 ,
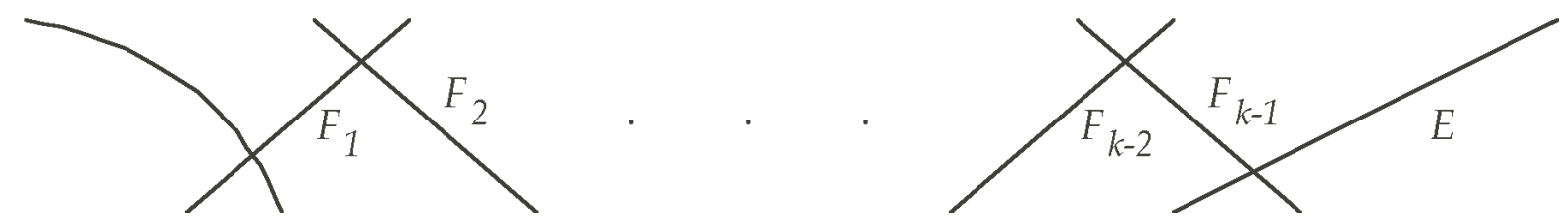

so that we can pass from $X$ to $X^{\prime}$ by $k$ successive contractions of exceptional curves of the first kind in smooth surfaces. Therefore it suffices to do the case $k=1$. But then the total transform of $C^{\prime}$ in $X$ is $C+E$, hence

$$
\left(C^{\prime} \cdot C^{\prime}\right)=(C \cdot C)+2(C \cdot E)+(E \cdot E)=(C \cdot C)+1=(C \cdot C)+\mu,
$$


as desired.

One consequence of $(4.1)$ is that $(C \cdot C) \leq 0$ when $g \geq 1$. In fact, by successively blowing down exceptional components we may pass from $f: X \rightarrow S$ to a family of semistable curves; Lemma (4.1) guarantees that at each step the self-intersection of $C$ does not decrease. We are thus reduced to the case when the fibers of $f$ are semi-stable. In this case that $(C \cdot C) \leq 0$ is due to Arakelov (cf. [1] or [14]). It is perhaps worth noticing that Arakelov's result is an almost immediate consequence of what has been proved so far. For instance, in case $g>0$, one of the standard proofs is to apply the index theorem on the surface $X$ to the triple of divisors consisting of $\omega_{f}, C$, and a fiber of $f$, and to use the fact that $\left(\omega_{f} \cdot \omega_{f}\right) \geq 0$. That this is the case, in turn, follows from (3.4) and the special case of (2.2) proved in the previous section.

We may now complete the proof of (2.2). As we have observed in section 2 , we may assume that the general fiber of $f$ is smooth. We set $C_{i}=\sigma_{i}(S)$. The proof will be divided into several subcases, depending on $g$ and $n$. Suppose $g>1$. In the previous section we have done the case $n=0$ : we assume then that $n>0$. We claim that (4.1) reduces us to the case $n=1$. In fact there is one of the sections $C_{i}$, say $C_{1}$, such that the family $f: X \rightarrow S$, together with the section $C_{1}$, is not generically a product. But then omitting $C_{n}$ and contracting the exceptional components that meet $C_{n}$ and only one of the remaining $C_{i}$ produces a non-isotrivial family $F^{\prime}$ of semi-stable $(n-1)$-pointed curves; for each $i$, denote by $C_{i}^{\prime}$ the image of $C_{i}$. Further contractions, which do not affect the degrees of $\lambda, \delta$, and $\psi$, yield a non-isotrivial family $F^{\prime \prime}$ of stable $(n-1)$-pointed curves. Now, by (4.1), the selfintersections of the $C_{i}^{\prime}$ are of the form

$$
\left(C_{i}^{\prime} \cdot C_{i}^{\prime}\right)=\left(C_{i} \cdot C_{i}\right)+\mu_{i}
$$

Notice also that, by construction,

$$
\mu_{n}=\sum_{i=1}^{n-1} \mu_{i}
$$

Thus

$$
\operatorname{deg} \psi_{F^{\prime \prime}}=\operatorname{deg} \psi_{F^{\prime}}=-\sum_{i=1}^{n-1}\left(C_{i}^{\prime} \cdot C_{i}^{\prime}\right)=-\sum_{i=1}^{n-1}\left(C_{i} \cdot C_{i}\right)-\mu_{n}=\operatorname{deg} \psi_{F}-\mu_{n}+\left(C_{n} \cdot C_{n}\right),
$$

while

$$
\operatorname{deg} \lambda_{F^{\prime \prime}}=\operatorname{deg} \lambda_{F^{\prime}}=\operatorname{deg} \lambda_{F}, \quad \operatorname{deg} \delta_{F^{\prime \prime}}=\operatorname{deg} \delta_{F^{\prime}}=\operatorname{deg} \delta_{F}-\mu_{n},
$$

so that

$$
\begin{aligned}
12 \operatorname{deg} \lambda_{F}-\operatorname{deg} \delta_{F}+\operatorname{deg} \psi_{F} & =12 \operatorname{deg} \lambda_{F^{\prime \prime}}-\operatorname{deg} \delta_{F^{\prime \prime}}+\operatorname{deg} \psi_{F^{\prime \prime}}-\left(C_{n} \cdot C_{n}\right) \\
& \geq 12 \operatorname{deg} \lambda_{F^{\prime \prime}}-\operatorname{deg} \delta_{F^{\prime \prime}}+\operatorname{deg} \psi_{F^{\prime \prime}} .
\end{aligned}
$$

By induction on $n$, this proves our contention. If $f: X \rightarrow S$ is not isotrivial we can go one step further and omit $C_{1}$ as well, reducing to the case $n=0$, which we settled in the previous section. The case when $f: X \rightarrow S$ is isotrivial can be dealt with in several 
ways. One which is in the spirit of the rest of the paper is as follows. Write $C$ for $C_{1}$ and consider the line bundle $L=\omega_{f}^{k}(C)$, where $k \geq 3$. Then $f: X \rightarrow S$, together with $L$, is a generically non-trivial family of polarized noded curves, and the hypotheses of (3.2) are satisfied. In our situation $\left(\omega_{f} \cdot \omega_{f}\right), \operatorname{deg} \lambda_{f}$, and $\operatorname{deg} \delta_{f}$ all vanish; hence the left-hand side of (3.3) is a polynomial in $h$ whose coefficients are rational multiples of

$$
\operatorname{deg} \psi_{f}=12 \operatorname{deg} \lambda_{f}-\operatorname{deg} \delta_{f}+\operatorname{deg} \psi_{f}
$$

Furthermore its leading term is $g \operatorname{deg} \psi_{f}\left(h^{2} / 2\right)$ so that, for large $h,(3.3)$ yields $(2.2)$ in our situation.

Now suppose $g=1$. Using Lemma (4.1), the same argument as for $g>1$ shows that it suffices to do the case $n=2$ when $f: X \rightarrow S$ is generically a product family, and the case $n=1$. Suppose first that $n=1$ and write $C$ for $C_{1}$. For $k \geq 3$ the line bundle $L=\mathcal{O}(k C)$ satisfies the hypotheses of (3.2), with $r=d=k$. Looking at the exact sequences

$$
0 \rightarrow f_{*}(\mathcal{O}((i-1) C)) \rightarrow f_{*}(\mathcal{O}(i C)) \rightarrow f_{*}\left(\mathcal{O}(i C)_{\mid C}\right) \rightarrow 0
$$

for $i>1$ one sees easily that

$$
\operatorname{deg}\left(f_{*} L\right)=-\frac{k^{2}+k-2}{2} \operatorname{deg} \psi_{f}
$$

so the left-hand side of (3.3) has coefficients which are linear combinations of $\operatorname{deg} \lambda_{f}$ and of $\operatorname{deg} \psi_{f}$ and leading term $k(k-2) \operatorname{deg} \psi_{f} h^{2}$. Since in the situation we are considering

$$
12 \operatorname{deg} \lambda_{f}-\operatorname{deg} \delta_{f}=0
$$

(cf. [12]), this proves (2.2) for $g=n=1$. When $n=2$ and $f: X \rightarrow S$ is generically a product family, the argument is similar. We notice first that $\operatorname{deg} \lambda_{f}=0$ and that, by (4.1),

$$
\operatorname{deg} \delta_{f}=-\left(C_{1} \cdot C_{1}\right)=-\left(C_{2} \cdot C_{2}\right)=\frac{1}{2} \operatorname{deg} \psi_{f} .
$$

Next set $L=\mathcal{O}\left(k C_{1}+k C_{2}\right)$, with $k \geq 2$. Arguing as for $n=1$ one sees that, for some positive $\alpha$,

$$
12 \operatorname{deg} \lambda_{f}-\operatorname{deg} \delta_{f}+\operatorname{deg} \psi_{f}=\frac{1}{2} \operatorname{deg} \psi_{f} \geq \alpha m(S) .
$$

It remains to discuss the case $g=0$. To begin with, notice that $\operatorname{deg} \lambda_{F}=0$. Let $T$ be the normalization of $S$. Then $f^{\prime}: X^{\prime}=X \times_{S} T \rightarrow T$, together with the sections $C_{i}^{\prime}=C_{i} \times_{S} T$, is a family of stable $n$-pointed curves of genus zero, which we denote by $G$. Notice that

$$
\operatorname{deg} \delta_{G}=\operatorname{deg} \delta_{F}, \quad \operatorname{deg} \psi_{G}=\operatorname{deg} \psi_{F} .
$$

Let $p_{1}, \ldots, p_{N}$ be the singular points of the fibers of $f^{\prime}$. Near $p_{j}, X^{\prime}$ is of the form $x y=t^{\mu_{j}}$, where $t$ is a local parameter on $T$. Moreover $p_{j}$ dissects the fiber on which it lies in two components, one of which is $\nu_{j}$-pointed and the other $\left(n-\nu_{j}\right)$-pointed, where $\nu_{j} \leq n / 2$. We shall need the following result. 
Lemma (4.2). $\operatorname{deg} \psi_{F}=-\sum_{i=1}^{n}\left(C_{i}^{\prime} \cdot C_{i}^{\prime}\right)=\sum_{j=1}^{N} \mu_{j} \nu_{j}\left(n-\nu_{j}\right)$

For a proof we refer to [4], Lemma (4.8). Since $\nu_{j} \geq 2$ for every $j$, and $\operatorname{deg} \delta_{F}=\sum \mu_{j}$, the lemma says in particular that $\operatorname{deg} \psi_{F} \geq 4 \operatorname{deg} \delta_{F}$, and hence also that

$$
12 \operatorname{deg} \lambda_{F}-\operatorname{deg} \delta_{F}+\operatorname{deg} \psi_{F} \geq 3 \operatorname{deg} \delta_{F}
$$

We now contract components of the fibers of $f^{\prime}: X^{\prime} \rightarrow T$ until we get a family $h: Y \rightarrow T$ which, together with the images of $C_{1}^{\prime}, C_{2}^{\prime}$, and $C_{3}^{\prime}$, is a family of stable 3-pointed curves of genus 0 , and hence a product family. For each $i$, we let $D_{i}$ be the image of $C_{i}^{\prime}$ in $Y$. Since $F$ is assumed not to be isotrivial, at least one among the $D_{i}$ with $i>3$, say $D_{4}$, is not a constant section of $h$, that is, comes from a non-constant map from $T$ to $\mathbb{P}^{1}$. As this map factors through $S$, its degree is at least equal to $m(S)$. This means that the intersection multiplicity of $D_{4}$ and $D_{i}$ is at least equal to $m(S)$ for $i=1,2,3$, and, as a consequence, that

$$
\operatorname{deg} \delta_{F} \geq 3 m(S)
$$

Taking into account (4.3), this proves (2.2) in the case under consideration. The proof of Theorem (2.2), and hence of the projectivity of $\bar{M}_{g, n}$, is now complete.

\section{References}

[1] S. Ju. Arakelov, Families of algebraic curves with fixed degeneracies, Math. U.S.S.R. Izvestija 35 (1971), 1277-1301.

[2] E. Arbarello, M. Cornalba, The Picard groups of the moduli spaces of curves, Topology 26 (1987), 153-171.

[3] M. Artin, Versal deformations and algebraic stacks, Inventiones Math. 27 (1974), 165-189.

[4] M. Cornalba, J. Harris, Divisor classes associated to families of stable varieties, with applications to the moduli space of curves, Ann. scient. Éc. Norm. Sup. (4) 21 (1988), 455-475.

[5] D. Gieseker, Lectures on moduli of curves, Bombay 1982.

[6] J. Harris, D. Mumford, On the Kodaira dimension of the moduli space of curves, Inventiones Math. 67 (1982), 23-86.

[7] R. Hartshorne, Ample subvarieties of algebraic varieties, Lecture Notes in Math. 156, Berlin-Heidelberg-New York 1970.

[8] F. Knudsen, D. Mumford, The projectivity of the moduli space of stable curves I, Math. Scand. 39 (1976), 19-55.

[9] F. Knudsen, The projectivity of the moduli space of stable curves,

II, Math. Scand. 52 (1983), 161-199.

III, Math. Scand. 52 (1983), 200-212. 
[10] J. Kollàr, Projectivity of complete moduli, J. Differential Geometry 32 (1990), 235268.

[11] D. Mumford, Geometric invariant theory, Berlin-Heidelberg 1965.

[12] D. Mumford, Stability of projective varieties, L'Ens. Math. 23 (1977), 39-110.

[13] H. Popp, Moduli theory and classification theory of algebraic varieties, Lecture Notes in Math. 620, Berlin-Heidelberg-New York 1977.

[14] L. Szpiro, Propriétés numériques du faisceau dualisant, Astérisque 86 (1981), 44-78.

[15] E. Viehweg, Weak positivity and the stability of certain Hilbert points,

I, Inventiones Math. 96 (1989), 639-667.

II, Inventiones Math. 101 (1990), 191-223.

III, Inventiones Math. 101 (1990), 521-543. 